\title{
Study of vitamins, antioxidant and trace elements on various spirometric parameters and quality of life in patients of bronchial asthma
}

\author{
Garima Adhaulia ${ }^{1 *}$, Suryakant ${ }^{2}$, Ajay Verma ${ }^{3}$, Praveen Katiyar ${ }^{4}$, A. K. Sachan ${ }^{5}$, Rajendra Nath ${ }^{6}$, R. K. \\ Dixit $^{7}$
}

\author{
${ }^{1}$ Resident, ${ }^{2}$ Professor and Head, ${ }^{3}$ Associate Professor, ${ }^{4}$ Assistant Professor, ${ }^{5-7}$ Professor,,${ }^{1,5-7}$ Dept. of Pharmacology \\ \&Therapeutics, ${ }^{2,3}$ Dept. of Respiratory Medicine, ${ }^{1-3,5-7}$ K.G.M.U., Lucknow, ${ }^{4}$ University Institute of Health Sciences, Kanpur, \\ Uttar Pradesh, India
}

*Corresponding Author: Garima Adhaulia

Email: drgarima.26@gmail.com

\begin{abstract}
Introduction: Imbalance between reactive oxygen species and antioxidant defence mechanism produce oxidative stress which further augments and aggravates airway inflammation in asthma. The objective of this study is to find out the effect of antioxidants, trace elements supplementation on pulmonary functions and quality of life in asthmatics.

Material and Methods: A prospective, interventional study was conducted in the department of Respiratory Medicine, K.G.M.U., Lucknow. 84 newly diagnosed patients of asthma were recruited. Patients were randomly allocated into two groups using lottery system. One group (A) were given only anti-asthmatic treatment while other group (B) received vitamin, antioxidant, trace element supplementation along with anti-asthmatic treatment. Spirometric parameters $\left(\mathrm{FEV}_{1} \%\right)$ and quality of life were assessed at baseline and at regular intervals and the results were compared between the groups.

Results: In our study, males (58.33\%) outnumbered females (41.67\%). Maximum number of cases were in the age group 18 $30(39.29 \%)$ followed by $31-40(25.00 \%)$. Mean age of patients was $35.9 \pm 13.64$ years. Mean \pm SD of $\mathrm{FEV}_{1} \%$ in group A was $72.3 \pm 2.7$ while in group B it was $72.7 \pm 2.9$, which showed that there was no statistically significant difference in spirometric parameters post treatment between the two groups $(\mathrm{p}=0.3734)$. Similarly, quality of life also showed insignificant results (total score, $\mathrm{p}=0.1141$ ).

Conclusion: From the results of our study, it was concluded that there was no additional benefit of vitamins, antioxidant, trace elements supplementation on pulmonary functions and quality of life in asthmatic patients.
\end{abstract}

Keywords: Asthma, Oxidative stress, Antioxidants, Quality of life, Spirometry.

\section{Introduction}

Bronchial asthma is a chronic inflammatory disease of the airways, characterized by hyper-responsiveness and airflow limitation leading to recurrent attacks of breathlessness, wheezing and disease exacerbation which varies in severity and frequency. ${ }^{1}$ Its burden on society is enormous and imposes negative impact on the clinical outcome and quality of life of patient.

According to Global Asthma Report (2018) asthma affects approximately 339.4 million people worldwide and the prevalence increases by $50 \%$ every decade. $^{2}$ The occurrence of asthma deaths are over $80 \%$ in low and lower - middle income countries. WHO estimate of asthmatic patients in India is 15-20 million. ${ }^{3}$ Globally prevalence of asthma increases by
$50 \%$ every decade and estimated to range $3-38 \%$ in children and $2-12 \%$ in adults. $^{4}$

Since it is disease with multiple etiologies, currently there is no definite cure for asthma, the disease can only be controlled and improved symptomatically with the help of medications. Inflammation of the bronchus is the hallmark of asthma and studies have shown that oxidative stress augment and aggravates airway inflammation. Imbalance between production of reactive oxygen species and antioxidant defence mechanism produce oxidative stress in the body. ${ }^{5}$

Environmental triggering factors make the respiratory tract susceptible to oxidative stress. Reactive oxygen species production as a result of oxidative stress causes inflammation which in turn 
initiate sequence of events leading to oxidant mediated cellular injury. Studies have shown that patients with asthma are deficient in antioxidants leading to oxidative stress burden and pulmonary dysfunction. ${ }^{4}$ Oxidative stress initiated in the airway of bronchial asthma patient because of exogenous and endogenous reactive species leads to epithelial cell injury, induction of transforming growth factor $\beta 1$ secretion from epithelial cells resulting in remodeling of airway. $^{5}$

Antioxidants keep the airway healthy by neutralizing the damaging effects of oxidative radicals. Enzymatic antioxidants such as superoxide dismutases, catalase, glutathione peroxidases require trace elements like $\mathrm{Cu}, \mathrm{Zn}$, Se to catalyze free radicals. Non-enzymatic antioxidants such as Vitamin A, E, C and a large group of flavenoids directly react with free radicals and reduce their potency. ${ }^{4}$ It has been hypothesized that essential elements may play an important roles in asthma genesis since they take part in oxidative stress reactions as cofactors of antioxidant enzymes.

Intracellular and extracellular fluid in lungs has vitamin $\mathrm{C}$ a free radical scavenger that protect against exogenous and endogenous free radicals. ${ }^{6}$ It is the most abundant antioxidant substance which contributes to regeneration of membrane bound oxidized vit $\mathrm{E}$ to function again and has an antihistaminic effect too. Vit $\mathrm{E}$ has a structural role in stablizing membrane. By reducing external attacks in the lung and neutralizing oxidants effects, antioxidants vitamins modulate the development of asthma and the impairment of pulmonary function. ${ }^{6}$

Trace elements such as $\mathrm{Cu}, \mathrm{Zn}$, Se have an important role in inflammation, oxidant /antioxidant imbalance and may further affect the disease status. $\mathrm{Zn}$ has beneficial roles in respiratory epithelium: as an antioxidant, anti-inflammatory agent, antiapoptotic agent, cofactor for DNA synthesis .Se has a crucial role in the inflammatory asthmatic process with the ability to affect the adhesion molecules expression. ${ }^{7}$

Severity of airflow limitation, its reversibility, variability and confirmation of asthma can be assessed by measurement of lung function. Spirometry $\left(\mathrm{FEV}_{1} \%\right)$ is the most commonly and widespread acceptance methods to assess airflow limitation. ${ }^{6}$
Antioxidant and trace element deficiencies seems to be important factors in this regard. There are no clinical trials producing concrete results suggestive of vitamins, antioxidants, trace elements role in clinical outlook of asthma.

\section{Objectives}

1. To describe demographic and health characteristics of patients receiving treatment for Bronchial asthma coming to OPD at the Department of Respiratory Medicine, K.G.M.U., Lucknow.

2. To assess and find out the relation between vitamins, antioxidant, trace elements supplementation and pulmonary functions at the Department of Respiratory Medicine, K.G.M.U., Lucknow.

3. To assess the improvement in spirometric parameters in patients of bronchial asthma after supplementation with vitamins, antioxidants and trace elements at the Department of Respiratory Medicine, K.G.M.U., Lucknow.

4. To assess the improvement in quality of life in patients of bronchial asthma after supplementation with vitamins, antioxidants and trace elements at the Department of Respiratory Medicine, K.G.M.U., Lucknow.

\section{Material and Methods}

The present study was an interventional study conducted in the Department of Pharmacology and Therapeutics, King George's Medical University, Lucknow in collaboration with Department of Respiratory Medicine OPD, King George's Medical University, Lucknow. The study was started only after getting the ethical approval from the Institutional Ethics Committee of King George's Medical University, Lucknow. 84 newly diagnosed patients of asthma were recruited for the study after fulfilling our inclusion exclusion criteria. Written informed consent was taken from the study subjects. The total duration of study was of 12 months.

\section{Inclusion and exclusion criteria Inclusion Criteria}

1. Newly diagnosed patients of asthma. 
2. Patients of either sex of age 18 to 60 years

3. Patients having no associated comorbidities

4. Mild to moderate asthma cases with $\mathrm{FEV}_{1}>80 \%$ and $60-80 \%, \mathrm{FEV}_{1} / \mathrm{FVC}$ ratio normal to reduced $5 \%$

\section{Exclusion criteria}

1. Unwilling patients and those who did not give consent for the study

2. Patients who are unable to give interview

3. Pregnancy / Breastfeeding

4. Patients with incomplete medical records.

5. Patients with other chronic disease

6. Terminally ill patients

7. Patients with concurrent major psychiatric illness and/or concurrent major medical illness.

8. Patients with severe asthma with $\mathrm{FEV}_{1}<60 \%$ and $\mathrm{FEV}_{1} / \mathrm{FVC}$ reduced $>5 \%$.

The patients attending Respiratory medicine OPD and are newly diagnosed with asthma were recruited for the study. The diagnosis of asthma was made by detailed history, clinical symptoms, physical examination, investigations like pulmonary function tests (PFTs) - spirometry, Absolute Eosinophilic Count (AEC) and serum IgE levels. Once the diagnosis of asthma was confirmed patients were randomly allocated into two groups using lottery system. Group A patients were given anti-asthmatic treatment only while Group B patients received vitamin, antioxidant trace elements supplementation along with antiasthmatic treatment. Record of any previous baseline investigations was also made. A Case Report From (CRF) was designed as per the study protocol. It included patients demographic details - patients name, age, sex, occupation, residence, OPD registration number, presenting complaints and their duration, smoking history, family history of asthma, any previous treatment history or current treatment, associated comorbid conditions (such as HTN, Diabetes, TB, AIDS, heart disease etc), investigations related to diagnosis such as pulmonary function tests, average eosinophilic count (AEC), serum IgE levels etc, drugs prescribed for asthma along with dosage, duration, frequency, route of administration. The patients were followed up at $2^{\text {nd }}$ week, $4^{\text {th }}$ week (1month), $12^{\text {th }}$ week ( 3 month) and 6 months to assess the outcomes such as improvement in spirometric parameters $\left(\mathrm{FEV}_{1}, \mathrm{FEV}_{1} / \mathrm{FVC}\right)$ and quality of life. Quality of life was filled and assessed with the help of questionnaire- mini asthma quality of life (MQLQ) questionnaire at the time of enrollment of the patient (at baseline) and at follow up visits by interview method. Total of 84 patients will be enrolled into the study. The enrolled patients will be divided randomly into 2 groups:

1. Group A (42) - anti asthmatic treatment only

2. Group B (42) - anti asthmatic treatment + intervention (vitamins, antioxidant, trace element combination capsule)

At each visit spirometric parameters will be done along with the questionnaire related to any new symptom and improvement in quality of life

\section{Statistical evaluation}

Continuous variables are expressed as mean + SD and pre post data gas been compared by student's $\mathrm{t}$ test. $\mathrm{P}$ values less than 0.05 will be considered as statistically significant.

\section{Results}

\section{Demographic and health characteristics}

84 patients were studied during the 12 months study period. The age group ranged from 18 years and above (Table 1). Maximum number of cases were in the age group 18-30 (39.29\%) followed by 31-40 (25.00\%). Majority $(64.29 \%)$ of the patients were in the age group of $18-40$ years. 
Table 1: Demographic characteristics of the patients

\begin{tabular}{|c|c|c|c|c|c|}
\hline Age (Years) & $\mathbf{1 8 - 3 0}$ & $\mathbf{3 1 - 4 0}$ & $\mathbf{4 1 - 5 0}$ & $\mathbf{5 1 - 6 0}$ & $\mathbf{6 1}$ \& Above \\
\hline $\begin{array}{c}\text { Male } \\
(\mathbf{N = 4 9 )}\end{array}$ & 18 & 14 & 9 & 5 & 3 \\
$\mathbf{N}(\%)$ & $(54.55)$ & $(66.67)$ & $(56.25)$ & $(62.50)$ & $(50.00)$ \\
\hline $\begin{array}{c}\text { Female } \\
(\mathbf{N = 3 5 )}\end{array}$ & 15 & 7 & 7 & 3 & 3 \\
$\mathbf{N}(\%)$ & $(45.45)$ & $(33.33)$ & $(43.75)$ & $(37.50)$ & $(50.00)$ \\
\hline Total & & & & & \\
$(\mathbf{N = 8 4})$ & 33 & 21 & 16 & 8 & 6 \\
$\mathbf{N}(\%)$ & $(39.29)$ & $(25.00)$ & $(19.05)$ & $(9.52)$ & $(7.14)$ \\
\hline
\end{tabular}

The total number of males in the study was 49 (58.33\%) and females were 35 (41.67\%) (Table 2).Mean age of patients was $35.9 \pm 13.64$ years

Table 2: Gender wise distribution of the patients

\begin{tabular}{|c|c|c|}
\hline Gender & $\mathbf{N}(\boldsymbol{\%})$ & Mean Age \pm SD (years) \\
\hline Male & $49(58.33 \%)$ & $35.62 \pm 13.17$ \\
\hline Female & $35(41.67 \%)$ & $36.45 \pm 14.39$ \\
\hline Total & $84(100 \%)$ & $35.97 \pm 13.64$ \\
\hline
\end{tabular}

\section{Post treatment Spirometric parameters between groups}

Post treatment mean $\pm \mathrm{SD}$ of $\mathrm{FEV}_{1} \%$ in group A was $72.3 \pm 2.7$ and in group B it was $72.7 \pm 2.9$ which showed that there was no significant difference in spirometric parameters between the two groups $(\mathrm{p}=0.3734)$.

Table 3: Showing the comparison of improvement in $\mathrm{FEV}_{1}$ value in between groups

\begin{tabular}{|l|c|c|}
\hline \multirow{2}{*}{ Group A (Anti asthmatic treatment) } & \multicolumn{2}{|c|}{ FEV $_{\mathbf{1 \%}}$} \\
\cline { 2 - 3 } & At end of therapy (mean \pm SD) & p value \\
\hline $\begin{array}{l}\text { Group B (Anti asthmatic treatment + vitamin } \\
\text { antioxidant trace elements supplementation) }\end{array}$ & $72.3 \pm 2.7$ & 0.3734 \\
\hline
\end{tabular}

Relation between vitamins, antioxidant, trace elements supplementation and pulmonary functions:

There was no significant improvement in $\mathrm{FEV}_{1 \%}$ between two groups $(\mathrm{p}=0.3734)$ which denotes that there was no additional benefit of giving vitamins, antioxidant and trace element supplementation on pulmonary functions and quality of life in asthmatic patients.

\section{Quality of Life}

\section{Mini asthma quality of life questionnaire (mini AQLQ) for Asthma}

Domains of health related to quality of life were expressed as four domains of the mini asthma quality of life questionnaire (mini AQLQ) containing 15 questions grouped under four domains - Symptoms, Activity limitation, Emotional function, Environmental stimuli. Mean score for each domain was calculated pre and post treatment. Total score was recorded as the average of the scores in 15 questions. For both the groups post treatment mean \pm SD value of MQLQ domains were compared shown in Table 4 . 
Table 4: Assessment of mini asthma quality of life questionnaire (MQLQ)

\begin{tabular}{|c|c|c|c|c|c|}
\hline \multirow{3}{*}{ MQLQ Domains } & \multirow{2}{*}{\multicolumn{2}{|c|}{$\begin{array}{l}\text { Group A } \\
\text { Post (42) }\end{array}$}} & \multirow{2}{*}{\multicolumn{2}{|c|}{$\begin{array}{l}\text { Group B } \\
\text { Post (42) }\end{array}$}} & \multirow[t]{3}{*}{ P value } \\
\hline & & & & & \\
\hline & Mean & SD & Mean & SD & \\
\hline Symptoms (5 items) & 5.86 & 0.72 & 5.94 & 0.75 & 0.4050 \\
\hline Activity Limitation (4 items) & 5.98 & 0.65 & 6.18 & 0.62 & 0.2459 \\
\hline Emotional Function (3 items) & 3.31 & 0.46 & 3.44 & 0.48 & 0.0541 \\
\hline Environmental Stimuli (3 items) & 3.43 & 0.74 & 3.53 & 0.61 & 0.5271 \\
\hline Total Score & 4.67 & 0.70 & 4.77 & 0.72 & 0.1141 \\
\hline
\end{tabular}

\section{Discussion}

Asthma is a chronic disease associated with inflammation of airways and immuno-allergic abnormality. Studies from past has provided, substantial evidence regarding the role of oxidative stress in the pathogenesis of chronic diseases such as asthma. But its confirmatory role is yet to be established. Studies also suggest that concurrent administration of antioxidants along with the current pharmacological treatment can be beneficial in the long term symptomatic improvement of the disease process.

In our study, the age group for asthma ranged from 18 years and above. Maximum number of cases were in the age group $18-30(39.47 \%)$ followed by $31-40$ $(25.44 \%)$. Majority $(64.91 \%)$ of the patients were in the age group of 18-40 years. According to our study, mean age of $35.9 \pm 13.64$ years favors the fact that disease was common in economically productive age group compared to other ages. Similar finding was shown in study done by Nitant $\mathrm{T}$ et $a{ }^{8},{ }^{8}$ in Gujarat which showed age group of $13-64$ years were more commonly affected with mean age of 39.8 years. However, finding in a study done by Micheal B et al, ${ }^{9}$ in Bengaluru, Karnataka showed 41-60 years as affected age group with mean age of 51.1 years.

Total number of males in the study was 49 (58.33\%) which out-numbered female 35(41.27\%). The results were comparable with other studies as in by More SR et al, ${ }^{10}$ in Maharashtra, showed that $54.5 \%$ were male and $45.5 \%$ were female. In a study by Arumugam V et al, ${ }^{11}$ in Dehradun, $64 \%$ were males and $36 \%$ were females and in other studies by Puranik A et $a l,{ }^{12}$ MM Rafeeqet al, ${ }^{13}$ Basavaraju et al, ${ }^{14}$ Micheal $\mathrm{B}$ et $\mathrm{al}^{9}$ showed similar trend of gender distribution. However, Rajathilagam $\mathrm{T}$ et $\mathrm{al}^{15}$ and Nitant $\mathrm{T}$ et $\mathrm{al}^{8}$ study showed female preponderance of the disease as compared to males. The reason for more males in our study may be attributed to more male to female ratio in Uttar Pradesh. ${ }^{16}$ Also, may be female patients are reluctant to utilize health care facilities unless they are critically ill.

In our study post treatment $\mathrm{FEV}_{1} \%$ in group $\mathrm{A}$ was $72.3 \pm 2.7$ and in group B was $72.7 \pm 2.9$ which showed that there was no significant difference in spirometric parameters between the two groups $(p=0.3734)$. In a similar case-controlled study conducted by picado $\mathrm{C}$ et al, ${ }^{17}$ no association was found between bronchial asthma and the intake or the circulating levels of micronutrients or antioxidants. In contrast, cross sectional study by Ochs Balcom ${ }^{18}$ showed the association of antioxidant nutrients and markers of oxidative stress with forced expiratory volume in the first second (FEV1\%). The study data included 218 persons with chronic airflow limitation showed that serum beta-cryptoxanthin, lutein/zeaxanthin, and retinol, and dietary beta-carotene, beta-cryptoxanthin, lutein/zeaxanthin, Vitamin $\mathrm{C}$ and lycopene were positively associated with $\mathrm{FEV}_{1} \%(\mathrm{P}<0.05)$.

The quality of life was analyzed using Mini asthma quality of life questionnaire (mini AQLQ) in asthma patients and it was found that total mean score post treatment in group A and B were $4.67 \pm 0.70$ and $4.77 \pm 0.72$ respectively. In our study statistically insignificant difference was found in the average total score post treatment $(p=0.1141)$. This signifies that addition of vitamin, antioxidant and trace element supplementation does not improve the quality of life of asthma patient. When compared between the various 
domains of mini AQLQ, mean score for individual domains also showed insignificant improvement post treatment.

\section{Conclusion}

In conclusion, we have not found any significant improvement by vitamin, antioxidant, trace element supplementation on pulmonary functions and quality of life in asthma patients.

\section{Acknowledgement}

I sincerely thanks almighty and my co-authors for their constant support and guidance throughout my work.

\section{Source of Funding}

Intramural Seed Grant Program(2017-18), K.G.M.U, Lucknow.

\section{Conflict of Interest}

None.

\section{References}

1. WHO. Asthma. WHO [Internet]. 2013 [cited 2019 Sep 9]; Available from: https://www.who.int/respiratory/asthma/en/

2. Global Asthma Network The Global Asthma Report [Internet]. 2018 [cited 2019 Sep 9]. Available from:http://www.globalasthmareport.org/Global\%20 Asthma\%20Report\%202018.pdf

3. World Health Organization (WHO). Fact sheet 2017 [internet]. Geneva:WHO; 2017. Available from http://www.who.int/respiratory/asthma/en/

4. Parvaiz A Koul, Dharmesh Patel. Indian guidelines for asthma: Adherence is the key. Lung India 2015;32(1).

5. Milos Jesenak, Maria Zelieskova, Eva Babusikova. Oxidative Stress and Bronchial Asthma in ChildrenCauses or Consequences?. Front Pediatr 2017;5:162.

6. Bishopp A, Sathyamurthy R. Biomarkers of oxidative stress and antioxidants in severe asthma A Prospective Case-Control Study. Ann Allergy Asthma Immunol 2017;118:445-51.

7. Akira Yamasaki, Yuji Kawasaki. Relationship between Oxidative Stress, Physical Activity, and Vitamin Intake in Patients with Asthma. Yonago Acta Med 2017;60:86-93.
8. Trivedi N. Prescribing pattern in patients of asthma visiting outpatient departments of a tertiary care hospital: a crosssectional, observational study. Int J Basic Clin Pharmacol 2017;6(3):587-91.

9. Michael B. Drug Utilization Evaluation Of Bronchial Asthma In Tertiary Care Hospital. World J Pharm Pharm Sci 2016;5(2):1075-91

10. More SR. A Prospective Study of Evaluation of Medical Prescriptions And Drug Utilization For Bronchial Asthma Patients At A Tertiary Care Hospital. IOSR J Dent Med Sci (IOSR-JDMS) 2017;16(11): 37-43

11. Arumugam $\mathrm{V}$ et al.Drug Utilization Assessment in Asthma Therapy through Prescription Monitoring at Dehradun Hospitals. Indian J Allergy Asthma Immunol 2008;22(1):15-8.

12. Puranik A, Totade S. Drug utilization study of bronchial asthma in adults at rural hospital. Int J Basic Clin Pharmacol 2019;8:120-7.

13. Rafeeq MM, Murad H. Evaluation of drug utilization pattern for patients of bronchial asthma in a government hospital of Saudi Arabia. Niger J Clin Pract 2017;20:1098105.

14. B.T. Jayadeva, P. Panchaksharimath. A retrospective study on drug utilization in patients with acute exacerbation of bronchial asthma in adults at a tertiary teaching hospital in Bengaluru. Egypt J Chest Dis Tuberc 2016;65:90-22

15. Rajathilagam T. Drug Utilisation Study In Bronchial Asthma In A Tertiary Care Hospital. Int J Pharm Appl 2012;3(2):297-305.

16. Lucknow District Population Census 2011-2019, Uttar Pradesh literacy sex ratio and density [Internet]. [cited 2019 Sep 21]. Available from: https://www.census2011.co.in/census/district/528-lucknow.

17. Picado C, Deulofeu R, Lleonart R, Agusti M, Mullol J, Quinto L et al. Dietary micronutrients/antioxidants and their relationship with bronchial asthma severity. Allergy 2001;56:43-9.

18. Ochs-Balcom HM, Grant BJ, Muti P. Antioxidants, oxidative stress, and pulmonary function in individuals diagnosed with asthma or COPD. Eur J Clin Nutr 2006;60:991-9.

How to cite this article: Adhaulia G, Suryakant, Verma A, Katiyar P, Sachan AK, Nath R, Dixit RK. Study of vitamins, antioxidant and trace elements on various spirometric parameters and quality of life in patients of bronchial asthma. Int $J$ Comprehensive Adv Pharmacol 2019;4(4):144-9. 